MKG-Chirurg 2020 $\cdot 13: 133-134$

https://doi.org/10.1007/s12285-020-00246-6

Online publiziert: 23. März 2020

(c) Springer Medizin Verlag $\mathrm{GmbH}$, ein Teil von Springer Nature 2020

\title{
Peter Stoll
}

Prof. Dr. Dr. P. Stoll \& Partner, Freiburg, Deutschland

\section{Nachruf auf Prof. Dr. med. Wilfried Schilli}

\author{
Ein Titan der Mund-, Kiefer- und \\ Gesichtschirurgie
}

Seine Vita umfassend wiederzugeben, Mit Prof. Dr. med. Wilfried Schilli (- Abb. 1) ist ein großer akademischer Lehrer und Visionär, dem nicht nur die deutschsprachige, sondern auch die internationale Mund-, Kiefer- und Gesichtschirurgie wesentliche Impulse $\mathrm{zu}$ verdanken hat, am 29. September 2019 im hohen Alter von 91 Jahren von uns gegangen.

Wenn dieser Nachruf persönlich ausfällt, sei mir dies bitte nachgesehen. Mit Wilfried Schilli habe ich nicht nur meinen ehemaligen „Chef", sondern einen väterlichen Freund und ein großes Vorbild verloren, bin ich doch bereits als Gymnasiast durch unsere über lange Jahre befreundeten Mütter in seinen „Dunstkreis" getreten.

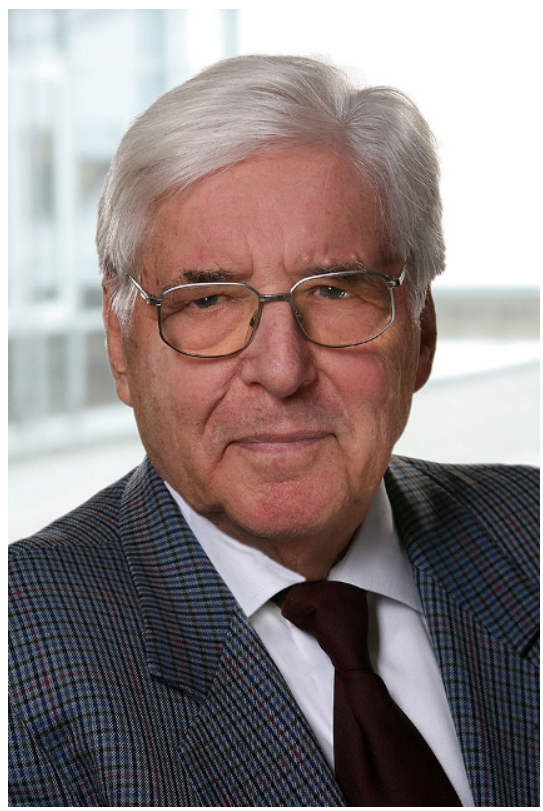

Abb. 1 ム Prof. Dr. med. Wilfried Schilli. (@ SIC invent $A G$ ) würde Bände füllen. Ich versuche es erst gar nicht.

Am 29. Mai 1928 in Offenburg geboren, wuchs Wilfried Schilli nach dem Umzug der Familie nach Freiburg in der Breisgaumetropole auf und besuchte dort das Rotteck-Gymnasium. Sein Vater Hermann war als Studienprofessor an der staatlichen Gewerbeschule für die Ausbildung von Zimmerleuten verantwortlich und erlangte später in Fachkreisen Berühmtheit als Buchautor, Gründer und Initiator des bekannten Schwarzwälder Freilichtmuseums Vogtsbauernhof in Gutach (Schwarzwaldbahn). Mit ihm mussten Wilfried Schilli und sein Bruder beinahe jedes Wochenende den Schwarzwald erwandern, um typische alte Schwarzwaldhäuser $\mathrm{zu}$ erkunden, was er - wie er viel später mir gegenüber bekannte - gar nicht lustig fand.

Nach dem Notabitur wurde er als Jugendlicher sofort zum Kriegsdienst eingezogen und begann nach Kriegsende eine Ausbildung zum Dentisten mit anschließendem Studium und Staatsexamen als Zahnarzt in Karlsruhe, nachdem das beabsichtigte Chemiestudium infolge der Kriegszerstörungen in Freiburg zunächst nicht möglich war. In der Zeit von 1949 bis 1955 folgte das Studium der Medizin in Freiburg. Als Medizinalassistent sammelte Wilfried Schilli Erfahrungen in innerer Medizin, Gynäkologie und Hals-Nasen-Ohren-Heilkunde. Bereits aus dieser Zeit datieren Freundschaften mit späteren Ordinarien. Ein fundiertes Basiswissen für seine spätere medizinische Karriere als Mund-, Kiefer- und Gesichts(MKG)Chirurg erarbeitete er sich anschließend als Assistent des renommierten Freiburger Pathologen Prof. Franz Büchner, mit dessen Sohn er befreundet war.

Schon immer faszinierte Wilfried Schilli die Kombination von Zahnmedizin und Chirurgie, sodass er sich 1958 dem zur damaligen Zeit noch Zahn-, Mund- und Kiefer(ZMK)-Chirurgie benannten Fachgebiet zuwandte und bei Prof. Josef Eschler, der in Personalunion den (damals noch) kieferchirurgischen und -orthopädischen Lehrstuhl am Freiburger Universitätsklinikum innehatte, seine Facharztausbildung begann. Die äußeren Bedingungen waren allerdings zu dieser Zeit noch ausgesprochen prekär, da die Klinik infolge von Bombeneinwirkung im Zweiten Weltkrieg vollständig zerstört und in den Freiburger Vorort Günterstal ausgelagert worden war. Dort waren die Behandlungsräume in einer ehemaligen Gastwirtschaft untergebracht, deren Decke zum 1. Obergeschoss aus statischen Gründen mit einem Baumstamm abgestützt wurde. Die Bettenstation befand sich im Günterstaler Waisenhaus, wo auch zahlreiche Kriegs- und Vertriebenenwaisen untergebracht waren.

Im Jahr 1961 konnte das neue Gebäude im Universitätsklinikum Freiburg bezogen werden, ein zu dieser Zeit kolossaler Neubau, der als der modernste Deutschlands galt. Im Gegensatz zu manchen anderen MKG-chirurgischen Universitätskliniken befand sich im selben Gebäude die Poliklinik. Dort hatte ich Wilfried Schilli, der seit 1963 schon habilitiert und Oberarzt war, als Pennäler 
besucht und nach dem rechten Weg gefragt. In dieser Zeit hatte er sich besonders der Spaltchirurgie zugewandt und durch seine zahlreichen Auslandsaufenthalte, v. a. auch einen 5-monatigen Arbeitsaufenthalt in Indien, einen enormen Schatz an Wissen und Erfahrung angeeignet. Gereist ist er immer gerne und polyglott war er auch.

Durch den unerwarteten und tragischen Tod JosefEschlers 1970 war der kieferchirurgische Lehrstuhl plötzlich verwaist und musste neu besetzt werden. Wilfried Schilli stand an dritter Stelle der Aspirantenliste. Nur wenige gaben ihm eine Chance. Eine glückliche Fügung ermöglichte dann doch die absolut unübliche Hausberufung.

Nicht nur die Freiburger Klinik profitierte dabei von seinem Talent, seiner Virtuosität als Redner, seiner ausgesprochenen Fähigkeit als Netzwerker und Verhandlungspartner und seiner raschen Auffassungsgabe mit dem Blick auf das Wesentliche. Darüber hinaus fielen ihm zahlreiche wichtige und ehrenvolle Aufgaben und Ämter zu. Davon seien nur einige auszugsweise erwähnt. So wurde er 1973 erster Vorsitzender der Arbeitsgemeinschaft für Kieferchirurgie der DGZMK. 1973 und 1974 wählte ihn die Medizinische Fakultät der Universität Freiburg zum Dekan. 1977 wurde er Jahrespräsident der DGMKG und richtete den Jahreskongress in Freiburg aus. Der Kongressausflug in den Kaiserstuhl und die Weinprobe in Bischoffingen sind wohl vielen älteren Kollegen noch in Erinnerung. 1983 übernahm er das Amt des Leitenden Ärztlichen Direktors am Freiburger Universitätsklinikum. Im gleichen Jahr richtete er als Tagungspräsident den Weltkongress der International Association of Oral and Maxillofacial Surgeons in Berlin aus.

Sein wissenschaftliches Evre umfasst mehr als 150 Publikationen und mehr als 10 Buch- und Handbuchbeiträge. Seit 1973 Mitglied der Arbeitsgemeinschaft für Osteosynthesefragen $(\mathrm{AO})$ hat er sich schon frühzeitig mit der funktionsstabilen Versorgung von Unterkiefer- und Mittelgesichtsfrakturen befasst und diese Methoden zusammen mit seinen Mitarbeitern auf unzähligen nationalen und internationalen Kongressen sowie Work- shops bekannt gemacht. Wilfried Schilli verband schon lange eine sehr gute und enge Bekanntschaft mit den Protagonisten der AO, sowohl mit den Wissenschaftlern als auch mit den Herstellern des Osteosynthesematerials. Als Mitglied der technischen Kommission der AO MF (MaxilloFacial) konnte er seine Ideen einbringen und umsetzen. Erwähnt sei in diesem Zusammenhang besonders die Entwicklung der „Freiburger Zuggurtungsplatte“. In der Spaltchirurgie galt sein besonderes Augenmerk der Rekonstruktion der Funktion der perioralen Muskulatur.

Die mehr oder weniger befriedigenden Resultate der konventionellen präprothetischen Chirurgie lenkten das Interesse von Wilfried Schilli schon bald auf das enorme Potenzial der oralen Implantologie. Dies war für einen Hochschullehrer und Ordinarius einer angesehenen deutschen Universitätsklinik durchaus ungewöhnlich, da sich die Implantologie zu dieser Zeit noch im "Rotlichtbezirk“ der Medizin bewegte und v. a. außeruniversitär betrieben wurde. Neue Entwicklungen hat er aber stets blitzschnell erkannt und sich an deren Spitze gestellt.

Seinem wissenschaftlichen Anspruch entsprechend wurde bereits 1975 der erste universitäre implantologische Workshop an der Freiburger Klinik angeboten, an dem ich als Medizinalassistent teilnehmen durfte. In der Folgezeit entwickelte sich die Implantologie rasant. Viele Impulse gingen dabei von der Freiburger Klinik aus.

Folgerichtig gehörte Wilfried Schilli zu den Gründungsmitgliedern des International Team of Implantology (ITI) und wurde 1992 zu dessen Präsidenten gewählt. Aus kleinsten Anfängen heraus umfasst das ITI heute weltweit mehr als 15.000 Mitglieder.

Auch nach seiner Emeritierung 1997 blieb Wilfried Schilli nicht untätig. Seinem Elan war der Ruhestand nicht angemessen. Er engagierte sich zusammen mit seinem Sohn Georg weiterhin in der Implantologie und wurde zum „businessman“ mit einer eigenen Firma. Und „busy“ war Wilfried Schilli eigentlich immer. So wurde er im Jahr 2000 von der Organisation Médecins du Monde gebeten, Ärzte der Welt als nationale Delegation in Deutschland zu gründen. Bis zu seinem Ausscheiden im Jahr 2010 trug er als Präsident der Ärzte der Welt zum ständigen Wachstum dieser Hilfsorganisation bei, die v.a. humanitäre Projekte unterstützt. Last, but not least soll sein jahrzehntelanges Engagement in der trinationalen Oberrheinischen Zahnärztegesellschaft nicht unterschlagen werden. Hier in der Metropolregion zwischen Straßburg, Freiburg und Basel zeigte sich Wilfried Schilli bis zuletzt als echter Europäer und Förderer des kollegialen Austauschs und der Verständigung über alle Grenzen hinaus.

Bei all seiner nationalen und internationalen Reputation hat Wilfried Schilli nie die Bodenhaftung verloren. Er sah sich immer als weltoffenen Badener. Während der 20 Jahre, die wir zusammenarbeiteten, habe ich ihn nie als überheblich erlebt. Als Chef war er für seine „Herren“ (Zitat), insbesondere in den letzten Jahren seines Wirkens, immer der Übervater, der es meisterlich verstand, sich mit guten Mitarbeitern zu umgeben, die der Klinik zu ihrem internationalen Ruf verhalfen. Einige seiner habilitierten Schüler wurden auf renommierte Lehrstühle berufen oder sie wurden Chef einer großen Klinik.

Auf unseren zahlreichen gemeinsamen Reisen hatten wir viele gute, nicht nur fachliche Gespräche. Wilfried Schilli war vielseitig interessiert. Gutem Essen und einem guten Tropfen war er auch nicht abgeneigt.

Sein Führungsstil in der Klinik war stets zeitgemäß, die Zügel hielt er locker in der Hand und förderte mannigfaltig die Ambitionen seiner Mitarbeiter, deren Kreativität er nach Kräften unterstützte. Dies fand nicht zuletzt Ausdruck in der Habilitation von 12 Mitarbeitern.

Wir trauern um diesen besonderen Menschen und Freund.

\section{Prof. Dr. Dr. Peter Stoll}

\section{Korrespondenzadresse}

Prof. Dr. med. Dr. med. dent. Peter Stoll

Prof. Dr. Dr. P. Stoll \& Partner

Wilhelmstraße 3, 79098 Freiburg, Deutschland prof.stoll@zahn-implantate-freiburg.de 